\title{
Land Capability Classification of Wadi Jerafi Basin, North Sinai Egypt
}

\author{
Emad A. Mahmoud ${ }^{1}$, Ahmed S. A. Sayed ${ }^{1}$ and Abdalsamad A.A. Aldabaa ${ }^{1}$
}

\begin{abstract}
The Egyptian Government pays a great attention nowadays to encourage the agricultural investment in Sinai Peninsula. The current study focused on wadi Jerafi which is one of the most promising areas in North Sinai Governorate. The goal of this study is to evaluate the land capability classification of Wadi Jerafi basin using two universal software's systems, namely as: MicroLEIS DSS (Cervatana Model) as semi-quantitative approach and Revised Storie Index as parametric approach. Accordingly, a grid soil survey was done through a total of 137 soil profiles. These soil profiles were investigated and sampled, then were chemically and physically analyzed. Based on soil variation in depth, gravel content and soil texture, seven soil mapping units (SMU) were delineated and evaluated to assess their agricultural capability. Cervatana Model classified the studied area into two capability classes; good capability (S2I) covering the largest area (63.25 \%) and marginal capability (S31) covering the lowest area $(36.75 \%)$. The most limiting factors are soil depth, gravel content, soil texture and/or salinity. On the other hand, Revised Storie Index (using Storie method equation) divided the land capability of the studied area into two classes unsuitable $(\mathrm{N})$ which covers of about 60.87 $\%$ of the total area and marginal suitable (S3) covering of about $39.13 \%$ of the total area. Another method of capability index calculation called Square Root Method was applied and therefore it distinguished three capability classes; moderately suitable (S2) covering $8.87 \%$ of the total area, marginal suitable (S3) covering $75.39 \%$ of the total area and unsuitable $(\mathrm{N})$ covering $15.74 \%$ of the total area. Accordingly, this paper recommend to implement Revised Storie Index as a parametric approach to evaluate the rate of each soil parameter and to use Square Root method to calculate the capability index of each mapping unit.
\end{abstract}

Keywords: Wadi Jerafi, Sinai, Egypt, Land capability, Cervatana Model, Storie Index

\section{INTRODUCTION}

Nowadays, the lack of arable land is one of the most constrains that is facing the developing countries such as Egypt. This problem has been magnified mainly due to number of variables; high population growth and decreasing soil fertility. As it is known, $95 \%$ of the total area of Egypt is considered as desert areas. On the other hand, the remaining area of Nile valley and Delta is under pressure whereas this arable land is being converted from agricultural use to urban and industrial uses. In addition to, the soil fertility of this area is continuously decreasing because of intensive agriculture per land unit to the extent that the arable land cannot compensate the lost necessary nutrients for plant growth. This situation needs to the intervention of governmental and private organizations in order to put alternative suitable solutions. One of these solutions is the agricultural extension especially in desert regions that should be taken place after executing comprehensive and integral land evaluation. Therefore, the Egyptian government has implemented many reclamation projects at different regions, (Abd-El Monsef et al., 2001) .One of areas being beheld by the Egyptian governorate for land reclamation is Sinai.

Land capability classification as a concept of land evaluation is one of the very remarkable issues in term of sustainable landuse, (Atalay, 2016). Many of land capability classification attempts have been set up and utilized predominately in USA, UK, and France as developed countries. Whatever land capability classification aim at grouping soils foremost on the basis of their ability to produce common cultivated crops and pasture plants without becoming progressively worse over long times, (Anderson, 1976). In general way, land capability classification expresses the suitability of soils for most types of field crops, (Rossiter, 1996). (Landon, 2014) reported that land capability classification, in general point of view, characterizes and evaluates land development units without putting in consideration the kind of use. Accordingly, some soils can be appropriate or convenient for specific crops and unsuitable for another's; in this manner precise selection of land utilization types is necessary. It could be known not only in terms of kind of crop production, but also how these crops are produced, (Van Ranst and Debaveye, 1991) and (Sys et al., 1991a).

The first trial for land capability classification system was proposed for classifying soils by (Klingebiel and Montgomery, 1961) through defining 8 classes. Class I for soils that have slight limitations that restrict their use. Class II for soils that have moderate limitations that restrict the choice of plants or that require moderate conservation practices. Class III for soils that have severe limitations that restrict the choice of plants or that require special conservation practices, or both. Class $I V$ for soils that have very severe

DOI: 10.21608/ASEJAIQJSAE.2019.27324

${ }^{1}$ Desert Research Center

Received December 09, 2018, Accepted February 12, 2019 
limitations that restrict the choice of plants or that require very careful management, or both. Class $V$ for soils that are subject to little or no erosion but have other limitations, impractical to remove, that restrict their use mainly to pasture, rangeland, forestland, or wildlife habitat. Class VI for soils that have severe limitations that make them generally unsuitable for cultivation and that restrict their use mainly to pasture, rangeland, forestland, or wildlife habitat. Class VII for soils that have very severe limitations that make them unsuitable for cultivation and that restrict their use mainly to grazing, forestland, or wildlife habitat. Class VIII for soils and miscellaneous areas that have limitations that preclude commercial plant production and that restrict their use to recreational purposes, wildlife habitat, watershed, or aesthetic purposes. A computer-based system for land capability classification called Cervatana model "General land capability" was created by (De la Rosa et al., 2004) defining 4 classes; (S1) land with excellent use capability, (S2) land with good use capability, (S3) land with moderate use capability, and $(\mathrm{N})$ Marginal or non-productive land. (O'Geen, 2008) had revised the Storie Index which is widely known and accepted method for rating soils for land use and productivity in California. This modified system rated land capability classes according to (Storie, 1978) where it categorized soil into 6 grades based on using the following equation to output the land capability classes:

Storie Index rating $=[($ Factor $\mathrm{A} / 100) \times($ Factor B/100) $\times($ Factor C/100 $) \times($ Factor X/100 $)] \times 100$

Where Factor $A$ is the effective soil depth in consideration, Factor $B$ is soil texture, Factor $C$ is slope, and Factor $X$ is containing drainage, alkalinity, fertility, acidity, erosion, and micro-relief.

The previous mentioned systems have been applied for evaluating different Egyptian areas. Sayed (2013) used USDA land capability system to evaluate the area extended along El-Hammam Canal, north west of Egypt whereby the studied area was classified into three classes VI, V, and VII. The same author applied Revised Storie Index which categorized the soils under investigation as Grade 2, 3, 4, 5 and 6 while by using MicroLEIS (Cervatana Model), the soils had recorded three classes S2, S3, and N. Aldabaa (2012) studied the land capability classification of some soils of wadi ElRayan and its environs, the study concluded that most of the investigated soils are not productive land or marginal with very few exceptions which are either moderate or good productive as indicated by Cervatana model. Revised Storie Index was applied in the same study and classified the soils of this area mainly as Grade 5 (very poor productive) with exceptional cases belongs to Grade 3 (fair productive) or Grade 4 (poor productive). Another study conducted the land capability classification using Storie Index for the soils along El-Salam Canal at north Sinai, was implemented by Abd-El Monsef et al., (2001). This study demonstrated that the soils along El-Salam Canal attained 5 capability classes ranging from Grade 2 to Grade 6. Gabour (1998) investigated the land capability classification at Northern Sinai Governorate, where the land capability classes found in this area were ranged from III to VI by applying (Klingebiel and Montgomery, 1961).

The current study is one of the continuous trails to evaluate the desert soils from agricultural point of view in order to explore the highly capable soils at the Egypt desert. Therefore, this study is aiming at evaluating the land capability using two types of land capability classification system, Cervatana model (MicroLEIS) and Revised Storie Index, to assess agricultural potential of an area at wadi Jerafi basin which is located at north east of Sinai and west of Egypt and Palestine border.

\section{MATERIALS AND METHODS Description of the study site}

The study area is a part of wadi Jerafi watershed which is located at the eastern portion of North Sinai governorate and situated between $34^{\circ} 34^{\prime} 26.7^{\prime \prime}$ to $34^{\circ}$ 43' 17.1" E and $29^{\circ} 54^{\prime} 47.4^{\prime \prime}$ to $30^{\circ} 5^{\prime} 28.4^{\prime \prime} \mathrm{N}$, adjacent to the international border between Egypt and Palestinian, Figure (1). The boundary of the study area, demarcated on 1:100000 a topographic map, occupies an area of about 58560 faddans. During the field study some locations of the whole study area were excluded as the following: First, about 19680 faddans $(33.61 \%$ of the total area) are covered by rough topography. Second, about 960 faddans ( $1.64 \%$ of the total area) is and old mine's field. Finally, about 1440 faddans (2.46 of the total area) is occupied by airport and military camp. The remaining area which has been actually studied is occupying 36480 faddans, representing about $62.29 \%$ of the total selected area).

Based on the metrological data as quoted from internal report (Desert Research Center, 2010), the climate of the studied area could be described as hot in summer and warm rainy in winter. The high mean of maximum and minimum temperature reached to 35 and $19{ }^{\circ} \mathrm{C}$, respectively during summer while they recorded as 16 and $2.5^{\circ} \mathrm{C}$, respectively during winter. The relative humidity ranged from $40-55 \%$ and the wind speed ranged from 13 to $18 \mathrm{~km} / \mathrm{hr}$ where the prevailing wind direction is South West in winter and North West in summer. The hours of solar radiation is relatively high where it ranged from 11.2 to 12.9 in summer and 9.0 to 
10.9 in winter leading to increase the evaporation rate which fluctuated between 3.31 on January and 8.81 on August. Evapotranspiration values were calculated using CROPWAT software, (Smith, 1992), where its values ranged from 3.76 to $9.16 \mathrm{~mm} /$ day.

In respect of geological setting (Figure 2), the surface exposures at wadi Jerafi Basin range from Early Cretaceous rocks (Malha Formation) to the quaternary (wadi fill deposits) as reported by EGPC (1987) and Desert Research Center (2010). Wadi Jerafi surface is covered by Eocene rocks especially the lower Eocene and middle Eocene represented by Egma and Mokattam Formation, respectively. Egma Formation consists of chalky limestone with flint bands and nodules at base and thin successive chert bands at top. Mokattam Formation consists of hard limestone rich in nummulites. On the other hand, the Quaternary rocks are represented by Holocene (wadi deposits), Pleistocene (fanglomerate and alluvial hammadah deposits), and Pliocene deposits (gravels and boulders of limestone). All of them are known as alluvial deposits which are composed of calcareous loamy sand and dark brown gravels forming the terraces of the dissecting wadis with varisized boulders of limestone, dolomite and chert. As for the geomorphic setting (Figure 2), wadi Jerafi basin is distinguished by different landforms which are tableland at North West, hilly area, low lands, and drainage lines or channels. The selected study area is covered by hilly area, low lands and drainage channels which are represented by soil profiles. The study revealed by Mahmoud et al. (2015) concluded that the groundwater could be existed at shallow depth in Quaternary deposits and fractured limestone.

\section{Soil samples collection and laboratory analyses}

After excluding the rough surface areas as well as the others inaccessible areas, 137 soil profiles on regular grid-based network $(1 \mathrm{~km} \mathrm{X} 1 \mathrm{~km})$ were investigated till $1.5 \mathrm{~m}$ or till the appearance of bed rock. As shown in Figure 2, the soil profiles are representing part of the drainage line and low land. Geologically, this part is covered by wadi deposits and Wasite formation which consists of gravel and boulders of limestone and chert. These soil profiles were described morphopedologically based on the criterion certified by Jahn et al. (2006). The soil's layers were sampled (about 339 soil samples) for carrying out the laboratory analyses to determine some chemical and physical properties. The soil texture analyses as well as gravel volume, soil water characteristics, electrical conductivity (EC), soil reaction $(\mathrm{pH})$, soil organic matter $(\mathrm{SOM})$, calcium carbonate, and cation exchangeable capacity were analyzed according to USDA (2004). Sodium exchangeable percent and sodium absorption ration were mathematically calculated according to Rashidi and Seilsepour (2008) and Al-Busaidi and Cookson (2003), respectively.

\section{Soil mapping units processing}

The soil mapping units were based on depicting the spatial distribution of the most uncorrectable limiting factors in the studied area which are soil depth, gravel content and soil texture (Figure 3A, 3B and 3C). They were automatically interpolated and reclassified according to Soil Science Division Staff ( 2017) and Schoeneberger (2012) using ArcGIS10.4.1, as shown in Table (1). After reclassification and coding the selected properties, they mathematically combined using PLUS spatial analyst tool, ArcGIS 10.4.1 to delineate the final soil mapping units, Table (2).

Table1 . Soil parameters used for delineating soil mapping units with reclassification codes

\begin{tabular}{ccccccccc}
\hline $\begin{array}{c}\text { Soil } \\
\text { property }\end{array}$ & Soil depth & \multicolumn{3}{c}{ Gravel content } & & Soil texture \\
\hline Interval & $0-50$ & $50-100$ & $\geq 100$ & $0-15$ & $15-40$ & $\geq 40$ & $\begin{array}{c}\text { Sand, } \\
\text { loamy } \\
\text { sand }\end{array}$ & $\begin{array}{c}\text { Sandy loam, loam, silt } \\
\text { loam, silt, clay loam, sandy } \\
\text { clay loam, silty clay loam }\end{array}$ \\
Description & shallow & $\begin{array}{c}\text { Moderately } \\
\text { deep }\end{array}$ & Deep & $\begin{array}{c}\text { Non- } \\
\text { gravelly }\end{array}$ & Gravelly & $\begin{array}{c}\text { Very } \\
\text { gravelly }\end{array}$ & $\begin{array}{c}\text { Sandy } \\
\text { soils }\end{array}$ & Loamy soils \\
Code & 100 & 200 & 300 & 10 & 20 & 30 & 1 & 2 \\
\hline
\end{tabular}


Table 2. Final soil mapping units based on PLUS spatial analyst tool, ArcGIS 10.4.1.

\begin{tabular}{ccccl}
\hline \multicolumn{2}{c}{ Soil properties reclassified code } & \multirow{2}{*}{ Combination code } & \multicolumn{1}{c}{ Soil mapping unit } \\
\hline Soil depth & Gravel content & Soil texture & & \\
\hline 100 & 20 & 1 & 121 & SMU01: Shallow, gravelly sandy soils \\
200 & 10 & 1 & 211 & SMU02: Moderately deep, non-gravel sandy soils \\
200 & 20 & 1 & 221 & SMU03: Moderately deep, gravelly sandy soils \\
200 & 30 & 1 & 231 & SMU04: Moderately deep, very gravelly sandy soils \\
300 & 20 & 1 & 321 & SMU05: Deep, gravelly sandy soils \\
300 & 30 & 1 & 331 & SMU06: Deep, very gravelly sandy soils \\
300 & 20 & 2 & 322 & SMU07: Deep, gravelly loamy soils \\
\hline
\end{tabular}

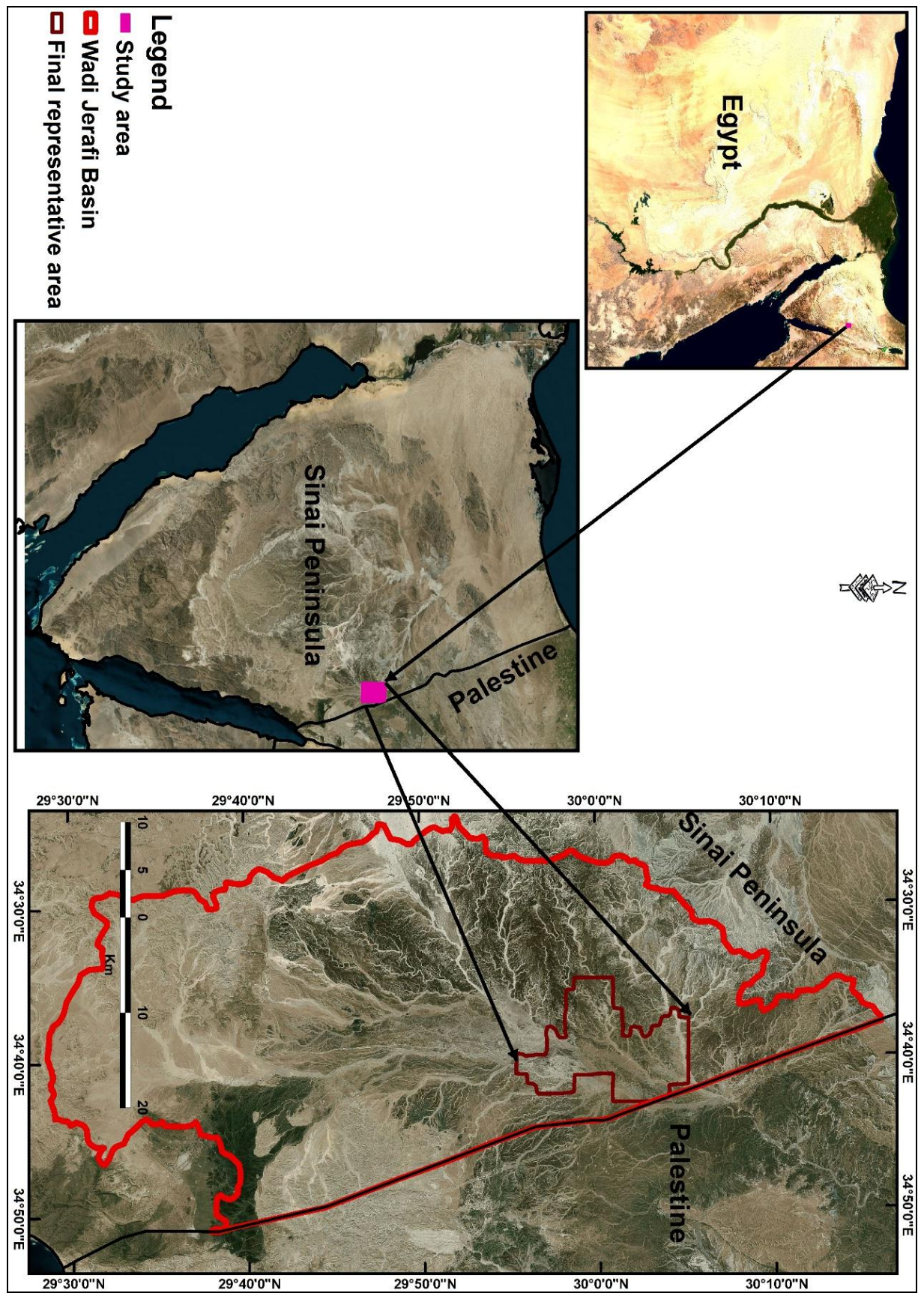

Figure 1. Location Map of the studied area showing Wadi Jerafi catchment area at Egyptian side 


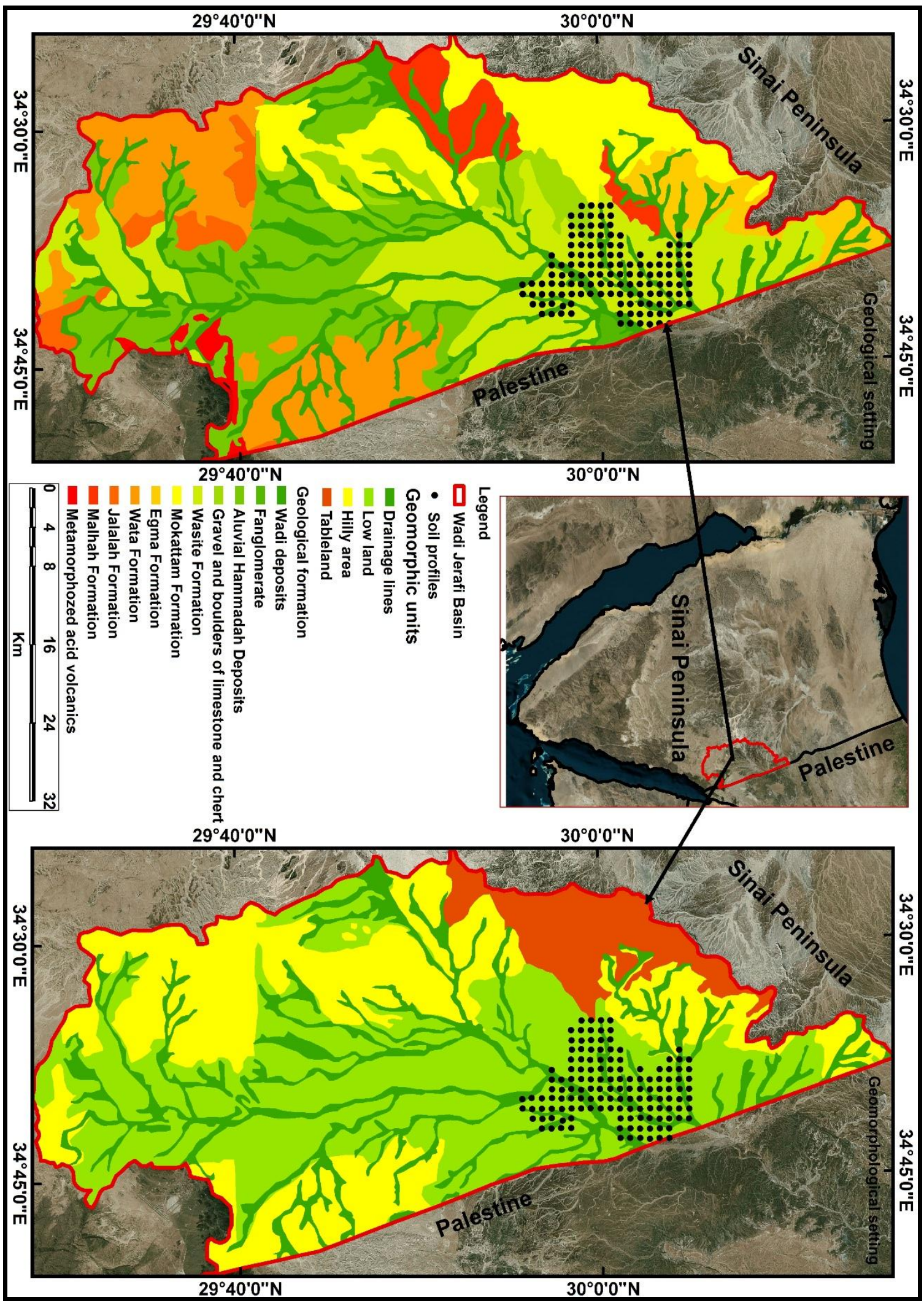

Figure 2. Geological and Geomorphological setting of wadi Jerafi at Egyptian side 


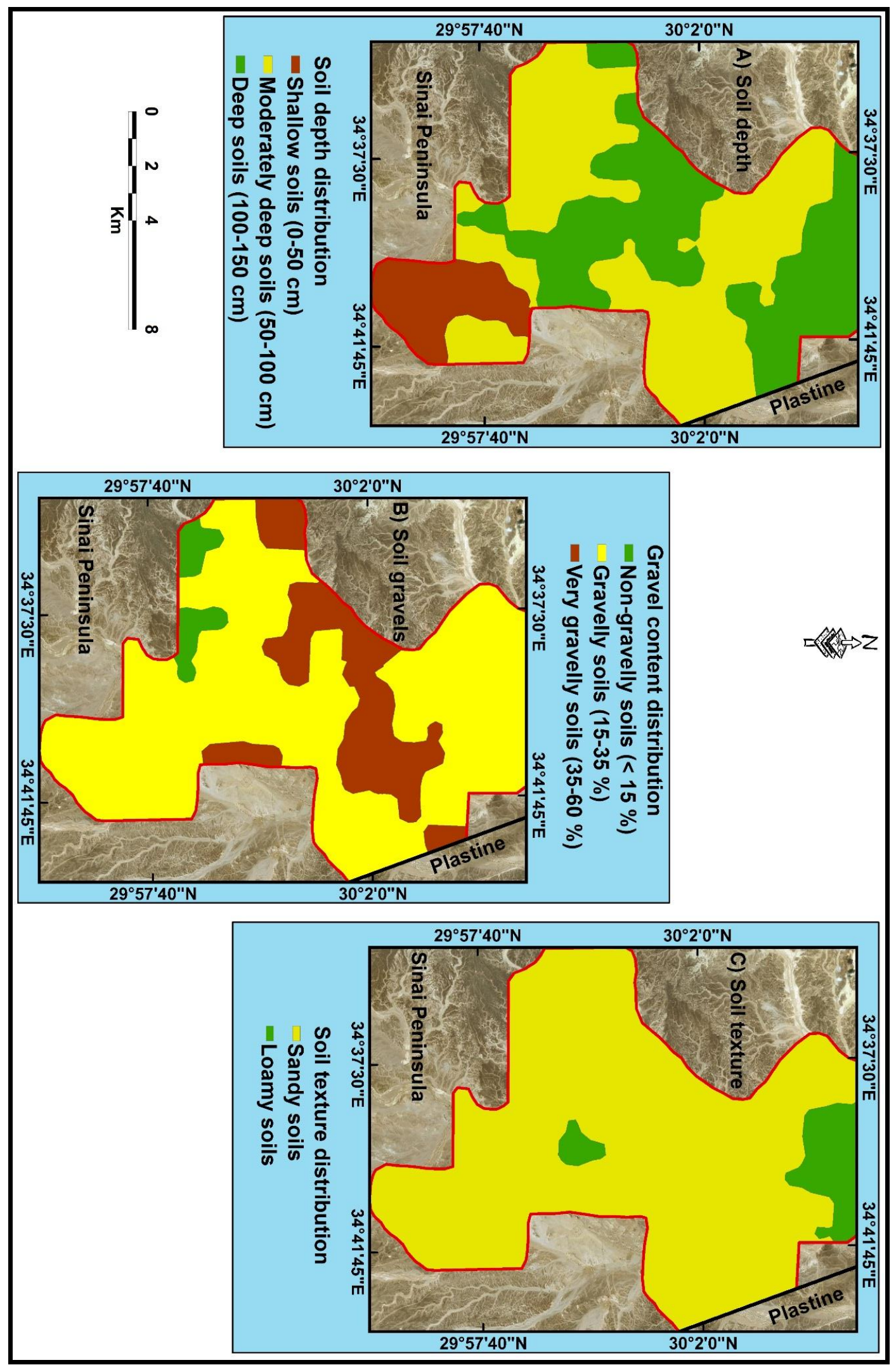

Figure 3.The main limiting (uncorrectable factors) used for delineating mapping units: (A) Soil depth (B) gravel content $(C)$ soil texture 


\section{Land capability classification \\ Semi-quantitative Methods}

For applying this method, General land capability (Cervatana) model was used and it is working interactively by comparing the values of the soil characteristics of each land unit to be appraised with the generalization levels established for each use capability class. The information and knowledge package in the structure of MicroLEIS DSS was implemented for organization, storage and reprocessing of databases for land capability classification, (De la Rosa et al., 2004). This model of land evaluation depends on evaluating all of topography factor (t), soil factor (l), Erosion risk factor (r) and bioclimatic deficiency factor (b). Both of erosion factor and bioclimatic deficiency factor are qualitative factors while topography factor and soil factor are quantitative. The processing using this model was done for each land unit to predict the general land capability.

\section{Parametric method}

The Revised Storie Index as a parametric method is widely adopted and accepted method of rating soils for landuse and productivity. It rates soils and assess the productivity based on the following four factors: Factor A, the degree of soil development; Factor B, surface texture; Facto C, slope; and Factor X, other soil and landscape properties including drainage, alkalinity, fertility, acidity, erosion and micro-relief. Rating of each factor mentioned above was scored according to Storie (1978) and O'Geen (2008). As for the final rate of each soil mapping units, this article used two different methods for calculation. The first one is called Storie method that apply the following equation, (O'Geen, 2008).

Storie Index rating $=($ Factor $\mathrm{A}) \times[($ Factor $\mathrm{B} / 100) \times$ $($ Factor $\mathrm{C} / 100) \times \ldots \ldots \ldots \ldots \ldots \ldots \ldots \ldots \ldots]$

The second method called Square Root Method (SRM) formulated by Khiddir et al. (1986) were used and its formula as following:

$\mathrm{SRM}$ rating $=$ Rate $_{\min } \times[($ Factor $\mathrm{A} / 100) \times($ Factor

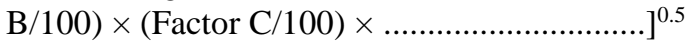

Based on the final score or rate of both methods, each land mapping unit was defined according to the following ranges or index values for the different suitability classes used by (Sys et al., 1991b): Very suitable (S1) with rate ranges from 75-100 \%; Moderately suitable (S2) with rate ranges from 50-75\%; marginally suitable (S3) with rate ranges from $25-50 \%$; Unsuitable $(\mathrm{N})$ with rate ranges from $0-25 \%$. All data were placed in an MS Excel spreadsheet for statistical analysis. First, the soil data of each soil profile were obtained by running the weighted average equation, namely; SUMPRODUCT. Then, the final data were split for each soil mapping unit and the basic descriptive analyses were done using XLSTAT plug-in software, (Addinsoft, 2017). The XLSTAT interface completely depends upon Microsoft Excel, whether for inputting data or for showing the results.

\section{RESULTS AND DISCUSSION}

As mentioned previously, the soil mapping units (SMU) were delineated based the weighted average of soil depth, gravel content and soil texture of each soil profiles. Accordingly, seven different soil mapping units as shown in Figure (4) were achieved and distinguished by some representative soil profiles Table (3) where statistically described and summarized as shown in Table (4).

In general, the surface slope of the studied area ranged widely from flat surface with zero percent as found in SMU03 and 07 to strongly sloping surface with $13.56 \%$ as found in SMU07. The soils reaction of the studied area ranged from natural $(\mathrm{pH} 7.0)$ as detected in SMU 01 to moderately alkaline $(\mathrm{pH} 8.43)$ as found in SMU 05. As for soil salinity, EC ranged widely from 0.1 to $1.78 \mathrm{dS} / \mathrm{m}$ as recorded in SMU03. Calcium carbonate content indicated that the soils of the studied area ranged widely from 10.31 (strongly calcareous) to $86.00 \%$ (extremely calcareous) as detected in SMU02 and SMU04, respectively. These high contents of calcium carbonate may be due to the origin of soil materials that are derived from chalky limestone. The soil sodicity measured by either SAR or ESP were recorded in low values in SMU 01, 05 and 06, while they are high in SMU02, 03, 04 and 07. Soil fertility was evaluated CEC and $\mathrm{OM}$ values which demonstrated that the studied area suffer from very poor fertility status. The mean values of the analyzed soil properties of each unit assessed through semi-quantitative method and parametric method for determining land capability by using MicroLEIS (Cervatana Model), (De la Rosa et al., 1992) and Storie Index model, (O'Geen, 2008), respectively.

\section{Semi-quantitative approach (Cervatana Model)}

The Cervatana model is one of the components of MicroLEIS DSS package which predicts the general landuse capability for a wide series of possible agricultural utilization, (De la Rosa et al., 2009). The required data could be grouped as biophysical factors: relief and soil as highlighted in Table (5) in addition to climate and current use. By implementing this model in regard to assessing the land capability classes, it was found that the area under consideration is characterized by two capability classes, namely good and marginally 
Table 3. Soil data of representative profiles of delineated soil mapping units (SMUs)

\begin{tabular}{|c|c|c|c|c|c|c|c|c|c|c|c|c|c|c|c|c|}
\hline \multirow{4}{*}{ Profile No. } & \multirow{3}{*}{$\begin{array}{l}\text { Depth } \\
\text { cm }\end{array}$} & \multirow{3}{*}{$\begin{array}{c}\text { Gravel } \\
\%\end{array}$} & \multicolumn{4}{|c|}{ Sand } & \multirow{3}{*}{$\begin{array}{c}\text { Silt } \\
\%\end{array}$} & \multirow{3}{*}{$\begin{array}{c}\text { Clay } \\
\%\end{array}$} & \multirow{3}{*}{ Texture } & \multirow{3}{*}{ pH } & \multirow{3}{*}{$\begin{array}{c}\text { EC } \\
\mathrm{dS} / \mathrm{m}\end{array}$} & \multirow{3}{*}{$\begin{array}{c}\mathrm{CaCO}_{3} \\
\%\end{array}$} & \multirow{3}{*}{$\begin{array}{c}\mathrm{CEC} \\
\mathrm{Cmol} / \mathrm{kg}\end{array}$} & \multirow{3}{*}{ SAR } & \multirow{3}{*}{ ESP\% } & \multirow{3}{*}{$\underset{\%}{O M}$} \\
\hline & & & & & $\%$ & & & & & & & & & & & \\
\hline & & & C.S & M.S & F.S & Total & & & & & & & & & & \\
\hline & \multicolumn{16}{|c|}{ SMU01: Shallow, gravelly sandy soils (3997.89 faddans) } \\
\hline 166 & $0--10$ & 38.50 & 35.22 & 30.63 & 34.15 & 100.00 & 0.00 & 0.00 & sand & 7.50 & 1.80 & 51.50 & 4.81 & 7.91 & 10.10 & 0.62 \\
\hline 199 & $0--18$ & 9.10 & 0.00 & 66.32 & 0.00 & 66.32 & 19.68 & 14.00 & sandy loam & 7.50 & 17.60 & 44.70 & 4.56 & 15.24 & 17.65 & 0.67 \\
\hline & $18--30$ & 12.20 & 11.10 & 25.06 & 63.84 & 100.00 & 0.00 & 0.00 & sand & 7.90 & 2.70 & 31.30 & 7.52 & 8.33 & 10.53 & 0.08 \\
\hline & $30--45$ & 60.60 & 62.96 & 22.11 & 14.93 & 100.00 & 0.00 & 0.00 & sand & 7.00 & 3.50 & 34.90 & 3.06 & 8.70 & 10.91 & 0.97 \\
\hline & & & SMI & $\mathrm{U} 02$ & Moder & rately d & leep, $n$ & hon-g & ${ }_{1}$ & y soils & s (174 & $45.46 \mathrm{f}$ & dans) & & & \\
\hline 152 & $0--30$ & 2.60 & 34.19 & 29.37 & 36.44 & 100.00 & 0.00 & 0.00 & sand & 7.50 & 0.50 & 32.50 & 5.02 & 7.31 & 9.48 & 0.58 \\
\hline & $30--80$ & 1.20 & 0.00 & 66.50 & 0.00 & 66.50 & 14.90 & 18.60 & sandy loam & 7.90 & 0.90 & 35.50 & 6.28 & 7.49 & 9.67 & 0.33 \\
\hline 163 & $0--50$ & 8.25 & 50.46 & 26.77 & 22.77 & 100.00 & 0.00 & 0.00 & sand & 8.00 & 0.30 & 32.60 & 3.78 & 7.22 & 9.38 & 0.83 \\
\hline & 50--70 & 26 & 55.48 & 22.99 & 21.53 & 100.00 & 0.00 & 0.00 & sand & 7.80 & 0.30 & 44.30 & 3.67 & 7.22 & 9.38 & 0.85 \\
\hline & & & 1U03: & $\mathrm{Mo}$ & lerately & y Deep & grave & elly s & $\cdot 1$ & 3141 & $12 f$ & ddans) & & & & \\
\hline 46 & $0--35$ & 15.00 & 20.98 & 23.52 & 55.60 & 100.10 & 0.00 & 0.00 & sand & 7.70 & 0.70 & 36.90 & .77 & 7.40 & 9.57 & 0.23 \\
\hline & $35--80$ & 42.90 & 50.75 & 22.99 & 26.26 & 100.00 & 0.00 & 0.00 & sand & 7.90 & 0.40 & 40.50 & 10 & 7.26 & 9.43 & 0.77 \\
\hline & 80--95 & 71.40 & 64.27 & 20.92 & 14.81 & 100.00 & 0.00 & 0.00 & sand & 7.50 & 2.00 & 42.20 & .05 & 8.01 & 10.20 & 0.97 \\
\hline 193 & $0--30$ & 12.27 & 17.88 & 19.94 & 52.18 & 100.00 & 0.00 & 0.00 & sand & 8.20 & 0.50 & 38.60 & 6.45 & 7.31 & 9.48 & 0.29 \\
\hline & $30--75$ & 58.00 & 44.33 & 30.66 & 25.01 & 100.00 & 0.00 & 0.00 & sand & 8.10 & 0.60 & 43.60 & & 7.36 & 9.53 & 0.79 \\
\hline & & SM & $\mathrm{J} 04$ & Mod & ately I & Deep, & ver & grav & sandy & s $(33$ & 321.2 & faddan: & & & & \\
\hline 44 & $0--60$ & 45.50 & 58.13 & 22.55 & 19.32 & 100.00 & 0.00 & 0.00 & sand & 7.90 & 0.80 & 43.70 & 3.46 & 7.45 & 9.62 & 0.89 \\
\hline & 60--90 & 46.70 & 80.87 & 13.58 & 5.55 & 100.00 & 0.00 & 0.00 & sand & 8.10 & 0.30 & 38.00 & 2.21 & 7.22 & 9.38 & 1.14 \\
\hline 70 & $0--40$ & 61.50 & 46.85 & 20.10 & 33.05 & 100.00 & 0.00 & 0.00 & sand & 7.70 & 0.40 & 44.80 & & 7.26 & 9.43 & 0.64 \\
\hline & $40--80$ & 37.10 & 40.90 & 10.04 & 9.69 & 60.63 & 20.17 & 19.20 & sandy loam & 7.90 & 0.50 & 42.70 & & 31 & 9.48 & 0.28 \\
\hline & 80--95 & 77.60 & 54.18 & 27.52 & 18.30 & 100.00 & 0.00 & 0.00 & sand & 7.70 & 0.10 & 49.30 & 7 & 7.12 & 9.29 & 0.91 \\
\hline & & & & $\mathrm{MU}$ & : Deep & $\mathrm{p}$, grav & elly sc & ndy & $S(0$ & $8 \mathrm{fad}$ & ddans) & & & & & \\
\hline 5 & $0--30$ & 19.10 & 18.52 & 19.97 & 61.51 & 100.00 & 0.00 & 0.00 & sand & 7.10 & 0.90 & 66.60 & & 7.49 & 9.67 & 0.13 \\
\hline & & & 41.53 & & 33.57 & & & & & 7.40 & & & & & 9.53 & 0.63 \\
\hline & 60--90 & 0.00 & 6.74 & 12.92 & 80.34 & 100.00 & 0.00 & 0.00 & sand & 7.40 & 1.00 & 71.70 & 9.02 & 7.54 & 9.72 & 0.22 \\
\hline & $90--150$ & 66.70 & 42.24 & 23.59 & 34.17 & 100.00 & 0.00 & 0.00 & sand & 7.40 & 0.70 & 77.40 & 4.82 & 7.40 & 9.57 & 0.62 \\
\hline 188 & $0--25$ & 4.40 & 10.04 & 25.46 & 64.50 & 100.00 & 0.00 & 0.00 & sand & 7.80 & 0.30 & 40.40 & .58 & 7.22 & 9.38 & 0.07 \\
\hline & $25--40$ & 21.10 & 54.58 & 32.24 & 13.18 & 100.00 & 0.00 & 0.00 & sand & 7.90 & 0.30 & 28.20 & & & 9.38 & 1.00 \\
\hline & $40--55$ & 15.40 & 36.41 & 26.52 & 37.07 & 100.00 & 0.00 & 0.00 & sand & 8.20 & 0.30 & 41.20 & 5.08 & 7.22 & 9.38 & 0.57 \\
\hline & $55--110$ & 24.00 & 55.07 & 30.14 & 14.79 & 100.00 & 0.00 & 0.00 & sand & 8.20 & 0.20 & 40.00 & 3.05 & 7.17 & 9.33 & 0.97 \\
\hline & & & & & 4U06: D & Deep, & very $g$ & ravell & sandy s & $(43$ & $40.6 \mathrm{f}$ & faddans) & & & & \\
\hline 85 & & & 19.52 & 17.05 & 63.43 & & 0.00 & 0.00 & sand & 7.30 & 3.00 & 49.30 & & 47 & & 0.09 \\
\hline & $20--35$ & 54.60 & 27.23 & 13.38 & 59.39 & 100.00 & 0.00 & 0.00 & sand & 7.20 & 5.90 & 37.50 & & 9.81 & 12.06 & 0.16 \\
\hline & 35--90 & 61.30 & 0.00 & 79.30 & 0.00 & 79.30 & 18.22 & 2.48 & loamy sand & 7.50 & 2.00 & 47.80 & 0.25 & 8.01 & 10.20 & 1.53 \\
\hline & $90--150$ & 40.00 & 0.00 & 82.51 & 0.00 & 82.51 & 10.00 & 7.49 & loamy sand & 7.90 & 0.20 & 32.00 & 2.13 & 7.17 & 9.33 & 1.16 \\
\hline 109 & $0--20$ & 0.00 & 16.44 & 11.15 & 72.41 & 100.00 & 0.00 & 0.00 & sand & 7.30 & 0.70 & 67.80 & 8.30 & 7.40 & 9.57 & 0.07 \\
\hline & $20--70$ & 75.00 & 29.86 & 18.28 & 51.86 & 100.00 & 0.00 & 0.00 & sand & 7.80 & 0.80 & 81.50 & 6.43 & 7.45 & 9.62 & 0.30 \\
\hline & $70--110$ & 0.00 & 10.78 & 14.39 & 74.83 & 100.00 & 0.00 & 0.00 & sand & 7.80 & 0.60 & & 8.52 & 7.36 & 9.53 & 0.12 \\
\hline & $110--150$ & 73.50 & 62.40 & 18.97 & 18.63 & 100.00 & 0.00 & 0.00 & sand & 7.80 & 0.60 & 85.80 & 3.40 & 7.36 & 9.53 & 0.90 \\
\hline & & & & & 1U07: & : Dee &, $\mathrm{gr}_{2}$ & eny & & 236. & $.05 \mathrm{fa}$ & & & & & \\
\hline 11 & $0--10$ & & 1.46 & 11.00 & 87.54 & 100.00 & 0.00 & 0.00 & sand & 8.10 & 0.60 & 73.20 & 9.67 & 7.36 & 9.53 & 0.35 \\
\hline & $10--40$ & 57.70 & 64.86 & 24.92 & 10.22 & 100.00 & 0.00 & 0.00 & sand & 8.00 & 0.50 & 40.30 & 2.64 & 7.31 & 9.48 & 1.06 \\
\hline & $40--70$ & 71.40 & 75.51 & 17.91 & 6.58 & 100.00 & 0.00 & 0.00 & sand & 7.90 & 0.70 & 40.70 & 2.30 & 7.40 & 9.57 & 1.12 \\
\hline & $70--110$ & 0.00 & 0.00 & 31.40 & 0.00 & 31.40 & 30.20 & 38.40 & clay loam & 8.10 & 2.30 & 40.10 & 13.69 & 8.14 & 10.34 & 1.15 \\
\hline 35 & $0--30$ & 19.20 & 33.68 & 19.95 & 46.37 & 100.00 & 0.00 & 0.00 & sand & 7.60 & 1.30 & 48.90 & 5.93 & 7.68 & 9.86 & 0.40 \\
\hline & $30--60$ & 18.80 & 50.84 & 26.23 & 22.93 & 100.00 & 0.00 & 0.00 & sand & 7.70 & 1.30 & 42.70 & 3.79 & 7.68 & 9.86 & 0.83 \\
\hline & 60--90 & 72.40 & 73.84 & 16.90 & 9.26 & 100.00 & 0.00 & 0.00 & sand & 8.00 & 0.90 & 41.60 & 2.55 & 7.49 & 9.67 & 1.07 \\
\hline & $90--140$ & 0.00 & 0.00 & 29.60 & 0.00 & 29.60 & 30.90 & 39.50 & clay loam & 8.20 & 0.70 & 46.90 & 14.10 & 7.40 & 9.57 & 1.23 \\
\hline
\end{tabular}


Table 4. Descriptive soil data of Mapping Units dominating in the studied area SMU01: Shallow, gravelly sandy soils (3998 faddans)

\begin{tabular}{|c|c|c|c|c|c|c|c|c|c|c|c|c|c|}
\hline Statistic & $\begin{array}{c}\text { Slope } \\
\% \\
\end{array}$ & $\begin{array}{c}\text { Depth } \\
\text { cm }\end{array}$ & $\begin{array}{c}\text { Gravel } \\
\% \\
\end{array}$ & $\begin{array}{c}\text { Sand } \\
\%\end{array}$ & $\begin{array}{c}\text { Silt } \\
\%\end{array}$ & $\begin{array}{c}\text { Clay } \\
\%\end{array}$ & pH & $\begin{array}{c}\text { EC } \\
\mathrm{dS} / \mathrm{m}\end{array}$ & $\begin{array}{c}\mathrm{CaCO}_{3} \\
\% \\
\end{array}$ & $\begin{array}{c}\text { CEC } \\
\mathrm{cmol} / \mathrm{kg}\end{array}$ & SAR & $\begin{array}{c}\text { ESP } \\
\% \\
\end{array}$ & $\begin{array}{c}\text { OM } \\
\%\end{array}$ \\
\hline Minimum & 1.72 & 10.00 & 18.50 & 86.53 & 0.00 & 0.00 & 7.00 & 0.20 & 19.80 & 4.75 & 7.17 & 9.33 & 0.07 \\
\hline Maximum & 5.91 & 45.00 & 39.84 & 100.00 & 7.87 & 5.60 & 8.38 & 8.93 & 56.10 & 7.94 & 11.22 & 13.51 & 0.63 \\
\hline Mean & 3.03 & 29.29 & 28.99 & 99.04 & 0.56 & 0.40 & 7.67 & 2.47 & 44.80 & 5.61 & 8.22 & 10.42 & 0.47 \\
\hline Variance (n-1) & 1.38 & 187.91 & 72.01 & 12.96 & 4.42 & 2.24 & 0.12 & 7.64 & 86.23 & 0.96 & 1.64 & 1.75 & 0.03 \\
\hline Standard deviation & 1.17 & 13.71 & 8.49 & 3.60 & 2.10 & 1.50 & 0.34 & 2.76 & 9.29 & 0.98 & 1.28 & 1.32 & 0.18 \\
\hline Standard error & 0.31 & 3.66 & 2.27 & 0.96 & 0.56 & 0.40 & 0.09 & 0.74 & 2.48 & 0.26 & 0.34 & 0.35 & 0.05 \\
\hline Skewness (Pearson) & 1.05 & -0.31 & 0.20 & -3.33 & 3.33 & 3.33 & -0.04 & 1.37 & -1.31 & 1.20 & 1.37 & 1.37 & -1.03 \\
\hline Kurtosis (Pearson) & 0.59 & -1.53 & -1.64 & 9.08 & 9.08 & 9.08 & 0.28 & 0.71 & 1.75 & 0.38 & 0.72 & 0.71 & -0.17 \\
\hline \multicolumn{14}{|c|}{ SMU 02:Moderately deep, non-gravelly sandy soils (1745 faddans) } \\
\hline Minimum & 2.07 & 70.00 & 0.00 & 82.66 & 0.00 & 0.00 & 7.09 & 0.40 & 10.30 & 4.46 & 7.26 & 9.43 & 0.22 \\
\hline Maximum & 5.11 & 95.00 & 7.93 & 100.00 & 8.44 & 10.50 & 7.68 & 17.56 & 82.52 & 7.78 & 15.22 & 17.63 & 0.69 \\
\hline Mean & 3.05 & 78.57 & 3.61 & 93.58 & 3.31 & 3.11 & 7.42 & 8.69 & 39.68 & 5.88 & 11.11 & 13.39 & 0.44 \\
\hline Variance $(\mathrm{n}-1)$ & 1.07 & 72.62 & 11.61 & 49.45 & 12.43 & 15.84 & 0.04 & 45.02 & 498.90 & 1.34 & 9.69 & 10.28 & 0.03 \\
\hline Standard deviation & 1.03 & 8.52 & 3.41 & 7.03 & 3.53 & 3.98 & 0.20 & 6.71 & 22.34 & 1.16 & 3.11 & 3.21 & 0.18 \\
\hline Standard error & 0.39 & 3.22 & 1.29 & 2.66 & 1.33 & 1.50 & 0.08 & 2.54 & 8.44 & 0.44 & 1.18 & 1.21 & 0.07 \\
\hline Skewness (Pearson) & 1.13 & 0.91 & 0.20 & -0.40 & 0.31 & 0.97 & -0.25 & -0.09 & 0.87 & 0.22 & -0.09 & -0.09 & 0.36 \\
\hline Kurtosis (Pearson) & 0.38 & 0.09 & -1.65 & -1.36 & -1.47 & -0.39 & -0.69 & -1.41 & 0.23 & -0.83 & -1.41 & -1.41 & -1.34 \\
\hline \multicolumn{14}{|c|}{ SMU03: Moderately Deep, gravelly sandy soils (13141 faddans) } \\
\hline Minimum & 0.00 & 50.00 & 15.24 & 79.30 & 0.00 & 0.00 & 7.00 & 0.10 & 19.20 & 1.31 & 7.12 & 9.29 & 0.11 \\
\hline Maximum & 7.86 & 95.00 & 39.71 & 100.00 & 15.40 & 7.25 & 8.25 & 18.78 & & 9.8 & 15.79 & 18.21 & 1.32 \\
\hline Mean & 3.12 & 73.17 & 27.90 & 97.92 & 1.22 & 0.86 & 7.53 & 4.53 & 43.33 & 5.77 & 9.18 & 11.40 & 0.54 \\
\hline Variance $(n-1)$ & 3.11 & 219.64 & 66.79 & 24.12 & 9.36 & 4.13 & 0.15 & 30.39 & 153.43 & 2.66 & 6.54 & 6.94 & 0.07 \\
\hline Standard deviation & 1.76 & 14.82 & 8.17 & 4.91 & 3.06 & 2.03 & 0.38 & 5.51 & 12.39 & 1.6 & 2.56 & 2.63 & 0.26 \\
\hline Standard error & 0.24 & 2.06 & 1.13 & 0.68 & 0.42 & 0.28 & 0.05 & 0.76 & 1.72 & 0.2 & 0.35 & 0.37 & 0.04 \\
\hline Skewness (Pearson) & 0.63 & -0.07 & -0.06 & -2.19 & 2.79 & 2.09 & 0.35 & 1.35 & 0.98 & 0.07 & 1.35 & 1.35 & 0.51 \\
\hline Kurtosis (Pearson) & -0.12 & -1.21 & -1.36 & 3.66 & 8.03 & 2.72 & -1.00 & 0.61 & 1.08 & 0.48 & 0.61 & 0.61 & 0.04 \\
\hline \multicolumn{14}{|c|}{ SMU 04: Moderately Deep, very gravelly sandy soils (3321 faddans) } \\
\hline Minimum & 0.06 & 50.00 & 42.69 & 83.43 & 0.00 & 0.00 & 7.06 & 0.20 & 18.67 & 3.05 & 7.17 & 9.33 & 0.34 \\
\hline Maximum & 4.91 & 95.00 & 64.70 & 100.00 & 8.94 & 8.39 & 8.32 & 15.64 & 86.0 & 8.1 & 14.33 & 16.71 & 0.97 \\
\hline Mean & 2.34 & 80.00 & 50.09 & 96.79 & 1.83 & 1.38 & 7.73 & 1.93 & 49.62 & 5.00 & 7.97 & 10.16 & 0.63 \\
\hline Variance $(n-1)$ & 2.49 & 160.71 & 38.09 & 36.99 & 11.27 & 8.53 & 0.11 & 15.48 & 310.99 & 1.45 & 3.33 & 3.53 & 0.03 \\
\hline Standard deviation & 1.58 & 12.68 & 6.17 & 6.08 & 3.36 & 2.92 & 0.33 & 3.94 & 17.63 & & 1.82 & 1.88 & 0.17 \\
\hline Standard error & 0.41 & 3.27 & 1.59 & 1.57 & 0.87 & 0.75 & 0.08 & 1.02 & 4.55 & 0.31 & 0.47 & 0.49 & 0.04 \\
\hline Skewness (Pearson) & 0.01 & -0.82 & 1.20 & -1.47 & 1.38 & 1.85 & -0.38 & 3.09 & 0.52 & 0.9 & 3.09 & 3.09 & 0.21 \\
\hline Kurtosis (Pearson) & -1.01 & -0.12 & 0.60 & 0.36 & 0.14 & 1.72 & -0.37 & 8.30 & -0.18 & 1.22 & 8.30 & 8.30 & -0.35 \\
\hline \multicolumn{14}{|c|}{ SMU05: Deep, gravelly sandy soils (6698 faddans) } \\
\hline Minimum & 1.29 & 100.00 & 15.67 & 87.54 & 0.00 & 0.00 & 7.03 & 0.17 & 4.45 & 2.8 & 7.16 & 9.32 & 0.34 \\
\hline Maximum & 7.12 & 150.00 & 39.23 & 100.00 & 6.68 & 6.07 & 8.43 & 6.95 & 72.40 & 10.40 & 10.30 & 12.56 & 1.02 \\
\hline Mean & 3.39 & 128.10 & 26.20 & 97.60 & 1.42 & 0.98 & 7.65 & 1.51 & 45.44 & 5.14 & 7.78 & 9.96 & 0.65 \\
\hline Variance $(n-1)$ & 3.19 & 396.19 & 49.12 & 20.20 & 6.86 & 4.09 & 0.13 & 4.14 & 268.83 & 2.91 & 0.89 & 0.95 & 0.03 \\
\hline Standard deviation & 1.79 & 19.90 & 7.01 & 4.49 & 2.62 & 2.02 & 0.36 & 2.03 & 16.40 & 1.71 & 0.94 & 0.97 & 0.18 \\
\hline Standard error & 0.39 & 4.34 & 1.53 & 0.98 & 0.57 & 0.44 & 0.08 & 0.44 & 3.58 & 0.37 & 0.21 & 0.21 & 0.04 \\
\hline Skewness (Pearson) & 0.98 & 0.15 & 0.61 & -1.37 & 1.26 & 1.80 & 0.01 & 1.66 & -0.39 & 1.38 & 1.67 & 1.66 & 0.43 \\
\hline Kurtosis (Pearson) & -0.28 & -1.81 & -0.88 & 0.06 & -0.36 & 1.56 & -0.43 & 1.55 & 0.26 & 2.46 & 1.56 & 1.54 & -0.37 \\
\hline \multicolumn{14}{|c|}{ SMU06: Deep, very gravelly sandy soils (4341 faddans) } \\
\hline Minimum & 1.20 & 100.00 & 41.73 & 85.41 & 0.00 & 0.00 & 7.27 & 0.22 & 33.14 & 2.65 & 7.18 & 9.34 & 0.37 \\
\hline Maximum & 11.37 & 150.00 & 60.93 & 100.00 & 10.68 & 5.60 & 8.25 & 4.07 & 79.60 & 6.43 & 8.96 & 11.18 & 1.05 \\
\hline Mean & 4.07 & 133.33 & 51.89 & 95.81 & 2.73 & 1.47 & 7.73 & 1.24 & 49.95 & 4.41 & 7.65 & 9.83 & 0.71 \\
\hline Variance $(n-1)$ & 5.36 & 366.67 & 49.32 & 33.70 & 15.00 & 4.04 & 0.08 & 1.80 & 282.97 & 1.69 & 0.38 & 0.41 & 0.06 \\
\hline Standard deviation & 2.32 & 19.15 & 7.02 & 5.81 & 3.87 & 2.01 & 0.28 & 1.34 & 16.82 & 1.30 & 0.62 & 0.64 & 0.25 \\
\hline Standard error & 0.60 & 4.94 & 1.81 & 1.50 & 1.00 & 0.52 & 0.07 & 0.35 & 4.34 & 0.34 & 0.16 & 0.17 & 0.06 \\
\hline Skewness (Pearson) & 2.10 & -0.37 & -0.31 & -0.77 & 0.87 & 0.82 & 0.13 & 1.14 & 0.69 & 0.22 & 1.14 & 1.13 & -0.18 \\
\hline Kurtosis (Pearson) & 4.83 & -1.54 & -1.49 & -1.19 & -0.88 & -0.89 & -0.99 & -0.23 & -1.05 & -1.48 & -0.23 & -0.25 & -1.50 \\
\hline \multicolumn{14}{|c|}{ SMU07: Deep, gravelly loamy soils (3236 faddans) } \\
\hline Minimum & 0.00 & 110.00 & 15.15 & 33.95 & 7.83 & 8.16 & 7.04 & 0.17 & 29.85 & 2.38 & 7.15 & 9.32 & 0.17 \\
\hline Maximum & 13.56 & 150.00 & 38.94 & 77.32 & 36.51 & 34.87 & 8.02 & 13.48 & 73.37 & 13.80 & 13.33 & 15.68 & 1.19 \\
\hline Mean & 3.70 & 146.15 & 24.44 & 63.08 & 17.22 & 19.69 & 7.48 & 4.19 & 50.61 & 8.20 & 9.02 & 11.24 & 0.81 \\
\hline Variance (n-1) & 18.50 & 125.64 & 60.17 & 269.12 & 81.51 & 82.61 & 0.14 & 22.71 & 193.63 & 12.24 & 4.89 & 5.19 & 0.10 \\
\hline Standard deviation & 4.30 & 11.21 & 7.76 & 16.40 & 9.03 & 9.09 & 0.37 & 4.77 & 13.92 & 3.50 & 2.21 & 2.28 & 0.31 \\
\hline Standard error & 1.19 & 3.11 & 2.15 & 4.55 & 2.50 & 2.52 & 0.10 & 1.32 & 3.86 & 0.97 & 0.61 & 0.63 & 0.09 \\
\hline Skewness (Pearson) & 1.49 & -2.89 & 0.79 & -1.03 & 1.15 & 0.49 & 0.14 & 1.14 & 0.42 & 0.00 & 1.14 & 1.14 & -0.66 \\
\hline Kurtosis (Pearson) & 0.93 & 6.79 & -0.64 & -0.61 & -0.04 & -1.26 & -1.55 & -0.44 & -1.00 & -0.92 & -0.44 & -0.44 & -0.45 \\
\hline
\end{tabular}




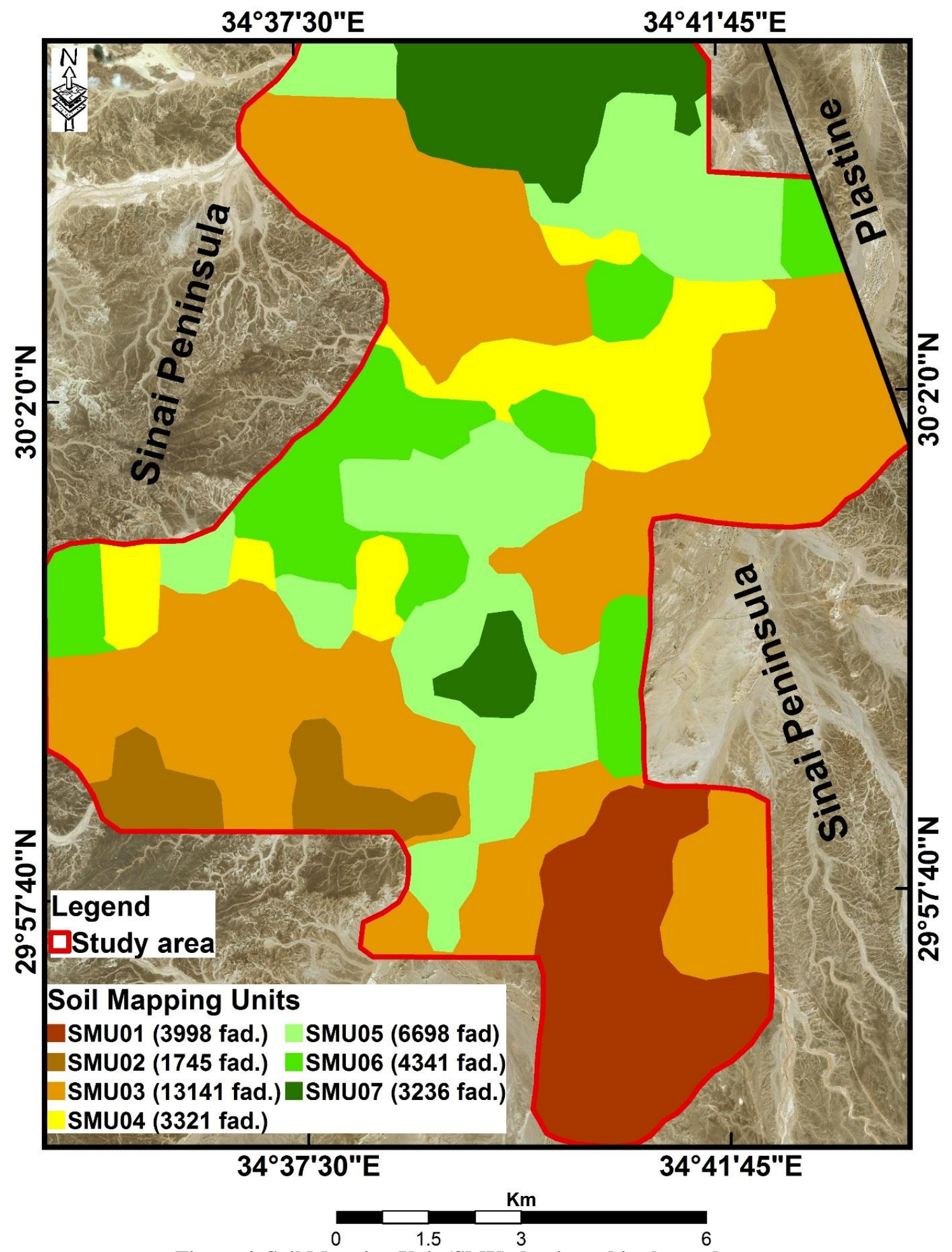

Figure 4. Soil Mapping Unit (SMU) dominated in the study area

SMU01: Shallow, gravelly sandy soils.

SMU03: Moderately deep, gravelly sandy soils SMU05: Deep, gravelly sandy soils SMU07: Deep, gravelly loamy soils
SMU02: Moderately deep, non-gravelly sandy soils SMU04: Moderately deep, very gravelly sandy soils SMU06: Deep, very gravelly sandy soils 


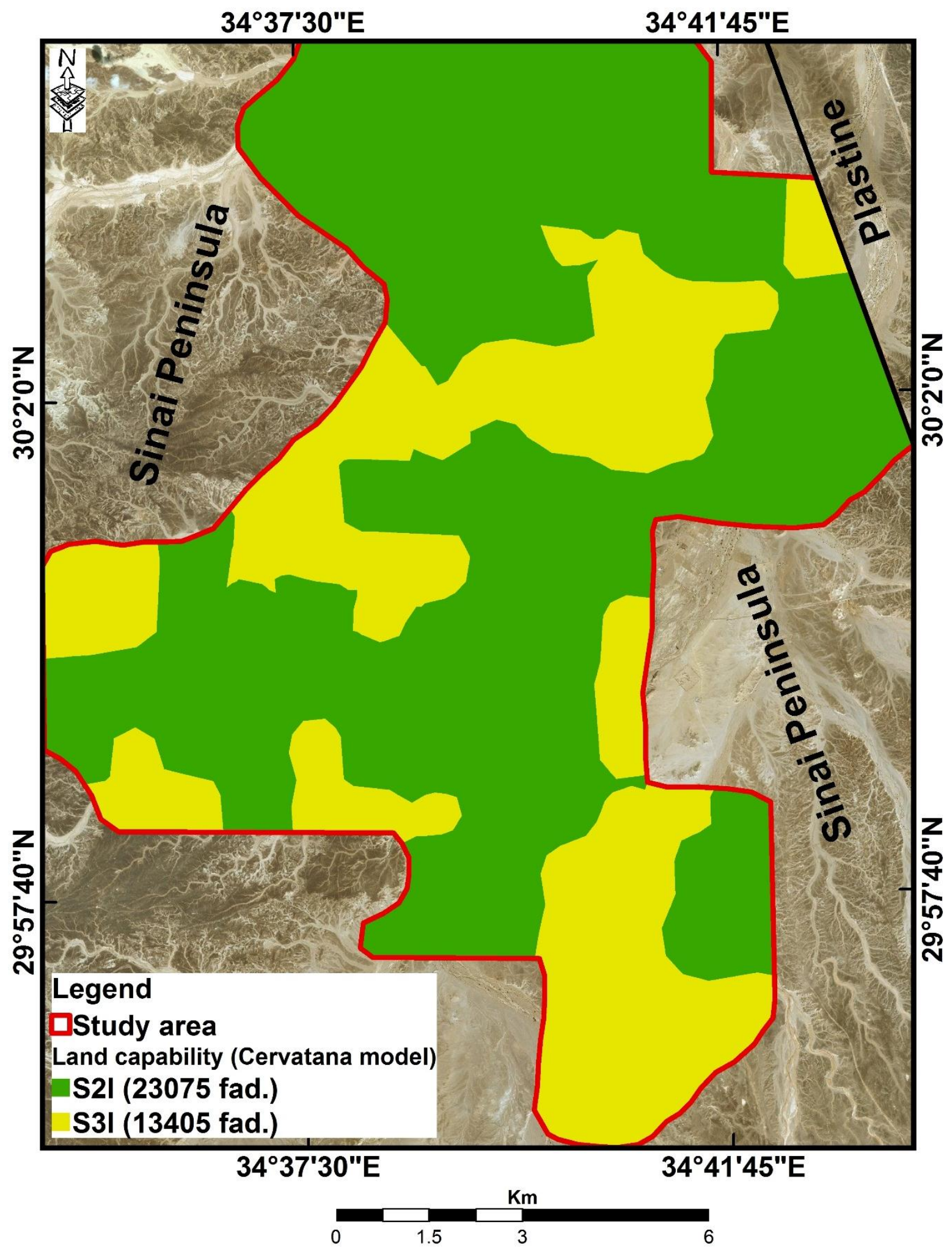

Figure 5. Land capability classes according to Cervatana Model (De La Rosa, 2004) 
Table 5. Some soil properties of soil mapping units and Cervatana model output

\begin{tabular}{|c|c|c|c|c|c|c|c|c|c|c|c|c|}
\hline SMU & $\begin{array}{c}\text { Slope } \\
\%\end{array}$ & $\begin{array}{l}\text { Depth } \\
\text { cm }\end{array}$ & $\begin{array}{c}\text { Gravel } \\
\% \\
\end{array}$ & Texture & pH & $\begin{array}{c}\text { EC } \\
\text { ds } / m\end{array}$ & $\mathrm{CaCO}_{3}$ & $\begin{array}{c}\begin{array}{c}\text { EPS } \\
\mathrm{cmol} / \mathrm{kg}\end{array} \\
\end{array}$ & SAR & $\begin{array}{c}\text { ESP } \\
\% \\
\end{array}$ & $\begin{array}{c}\text { OM } \\
\%\end{array}$ & Classes" \\
\hline SMU01 & 3.03 & 29.29 & 28.99 & Sand & 7.67 & 2.47 & 44.80 & 5.61 & 8.22 & 10.42 & 0.47 & S31 \\
\hline SMU02 & 3.05 & 78.57 & 3.61 & Sand & 7.42 & 8.69 & 39.68 & 5.88 & 11.11 & 13.39 & 0.44 & S31 \\
\hline SMU03 & 3.12 & 73.17 & 27.90 & Sand & 7.53 & 4.53 & 43.33 & 5.77 & 9.18 & 11.40 & 0.54 & S21 \\
\hline SMU04 & 2.34 & 80.00 & 50.09 & Sand & 7.73 & 1.93 & 49.62 & 5.00 & 7.97 & 10.16 & 0.63 & S31 \\
\hline SMU05 & 3.39 & 128.10 & 26.20 & Sand & 7.65 & 1.51 & 45.44 & 5.14 & 7.78 & 9.96 & 0.65 & S21 \\
\hline SMU06 & 4.07 & 133.33 & 51.89 & Sand & 7.73 & 1.24 & 49.95 & 4.41 & 7.65 & 9.83 & 0.71 & S31 \\
\hline \multirow[t]{2}{*}{ SMU07 } & 3.70 & 146.15 & 24.44 & Sandy loam & 7.48 & 4.19 & 50.61 & 8.20 & 9.02 & 11.24 & 0.81 & S21 \\
\hline & \multicolumn{3}{|c|}{ SMU: Soil Mapping Units } & Classes* $^{*}$ & \multicolumn{8}{|c|}{ Classes of land capability classification (Cervatana Model) } \\
\hline $\mathrm{S} 2$ & \multicolumn{3}{|c|}{ Good suitable } & $\mathrm{S} 2$ & Mc & erately & uitable & 1 & & \multicolumn{3}{|c|}{ Soil limitations } \\
\hline
\end{tabular}

land capability as shown in Figure (5) and Table (5). Good suitable (S21) which represented SMU03, 05 and 07 covers an area of about 23075 faddans $(63.25 \%)$ with soil limitations related to soil depth, gravel content and/or soil texture having different severity level. On contrary, SMU01, 02, 04 and 06 are represented by Marginally suitable (S31) which occupies an area of about 13405 faddans $(36.75 \%)$. The limiting factors that lower the land capability classes of these mapping units are soil depth, gravel, texture, soil salinity and/or slope.

\section{Parametric approach (Revised Storie Index)}

The Storie index is a semi-quantitative method for assessing potential land productivity by multiplying soil factors rates. The most ideal circumstances with regarding to each factor are rated at $100 \%$. Hence, the original Storie index has been mostly used in California and in order to apply it outside of this region and to reduce the subjectively innate to the original Storie index method, a Revised Storie Index was developed, (O'Geen, 2008). Accordingly, by applying Revised Storie Index with multiplying equation, two different capability classes were established in the studied area Table (6) and Figure (6). First class is Marginally suitable (S3) represented SMU01, 02, 03, 04 and 6 covering an area of about 14275 faddans (39.13\%). It is concluded that gravel content and soil texture are the main limiting factor $s$ of this class. The second class is Unsuitable (N) represented SMU05 and 07 covering an area of about 22205 faddans $(60.87 \%)$. Herein, soil depth and soil texture are the major limiting factors and in some cases gravel content and erosion were added as limiting factor of this capability classes
Applying the equation of Square Root method, the final capability index as shown Figure (7) and Table (5) was equaled with what were found in SMU01 and 02 as Unsuitable soils $(\mathrm{N})$ covering an area of about 5743 faddans $(15.74 \%)$, while it maximized the rates of SMU03, 04 and 06 were alleviated from Unsuitable (N) assessed by Storie method equation to Marginally suitable (S3) covering as area of about 27501 faddans $(75.39 \%)$, moreover the capability classes of SMU07 was maximized from Marginally suitable (S3) assessed by Storie method equation to Moderately suitable (S2) covering an area of about 3236 faddans $(8.87 \%)$. By comparing the applied of Revised Storie Index with Cervatana Model, the study found that Cervatana Model as a semi-quantitative method is not recommended to be applied under the Egyptian desert land condition where some characteristics are descriptive. On the other hand, Revised Storie Index is a recommended tool to evaluate the soil parameters according to the setup equation for each parameter. On contrary, while assessing the net value of land capability index of the soil unit, it is preferable to use the equation of Square Root Method rather than the equation of Storie Method.

It is found that the equation of the Storie Method minimized the final soil rate. Whatever, Egypt is in dire need to increase agricultural products to meet the needs of the growing population. Therefore, if the result of the Storie Method applied, a huge area especially in the studied area and generally in Egypt will be neglected. Oppositely, Applied the equation formulated by (Khiddir et al., 1986), the agricultural land utilization should be sensitively implemented in order to prevent land degradation. 


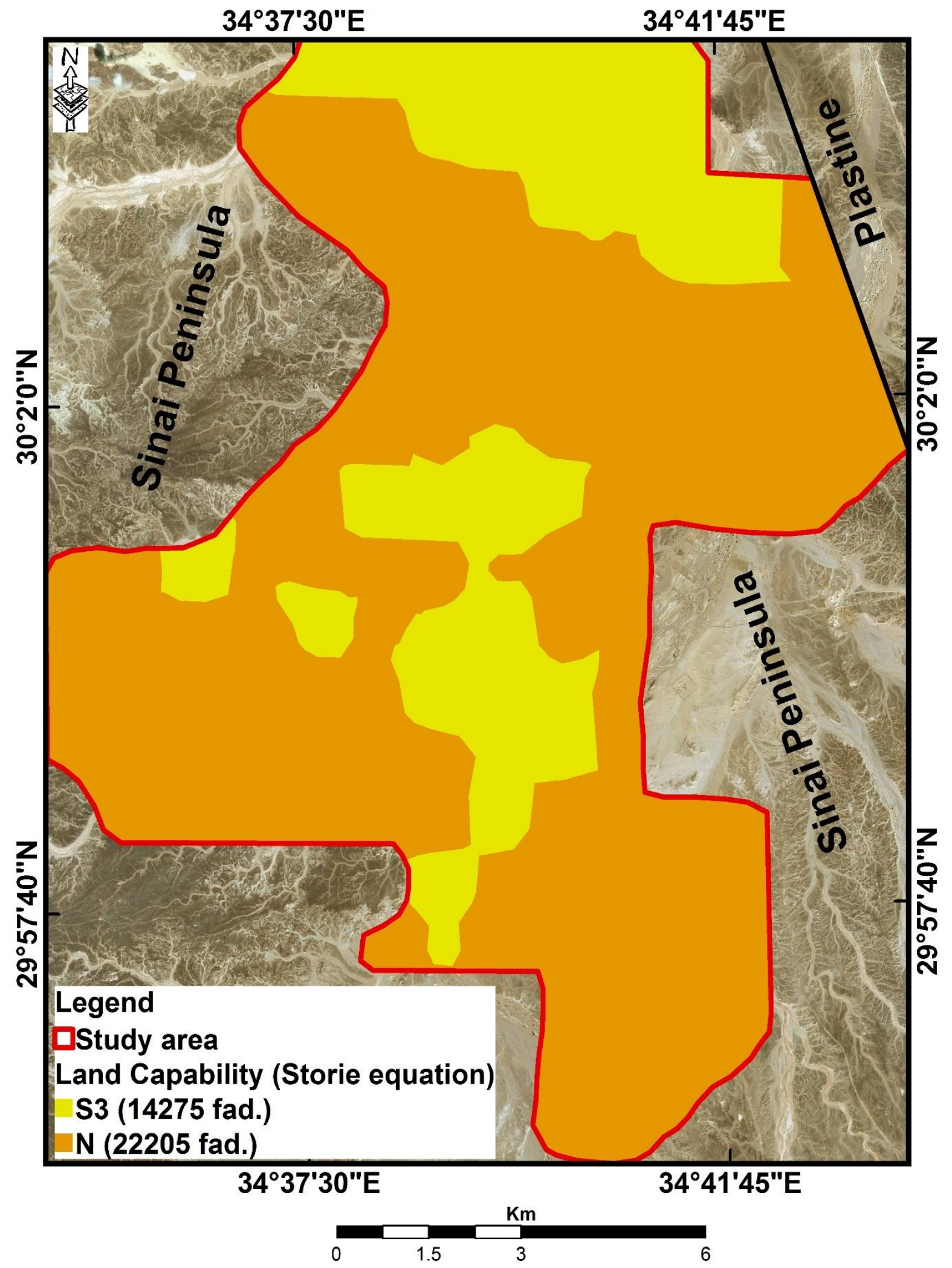

Figure 6. Land capability classes according to Storie method equation (O'Geen, 2008) 


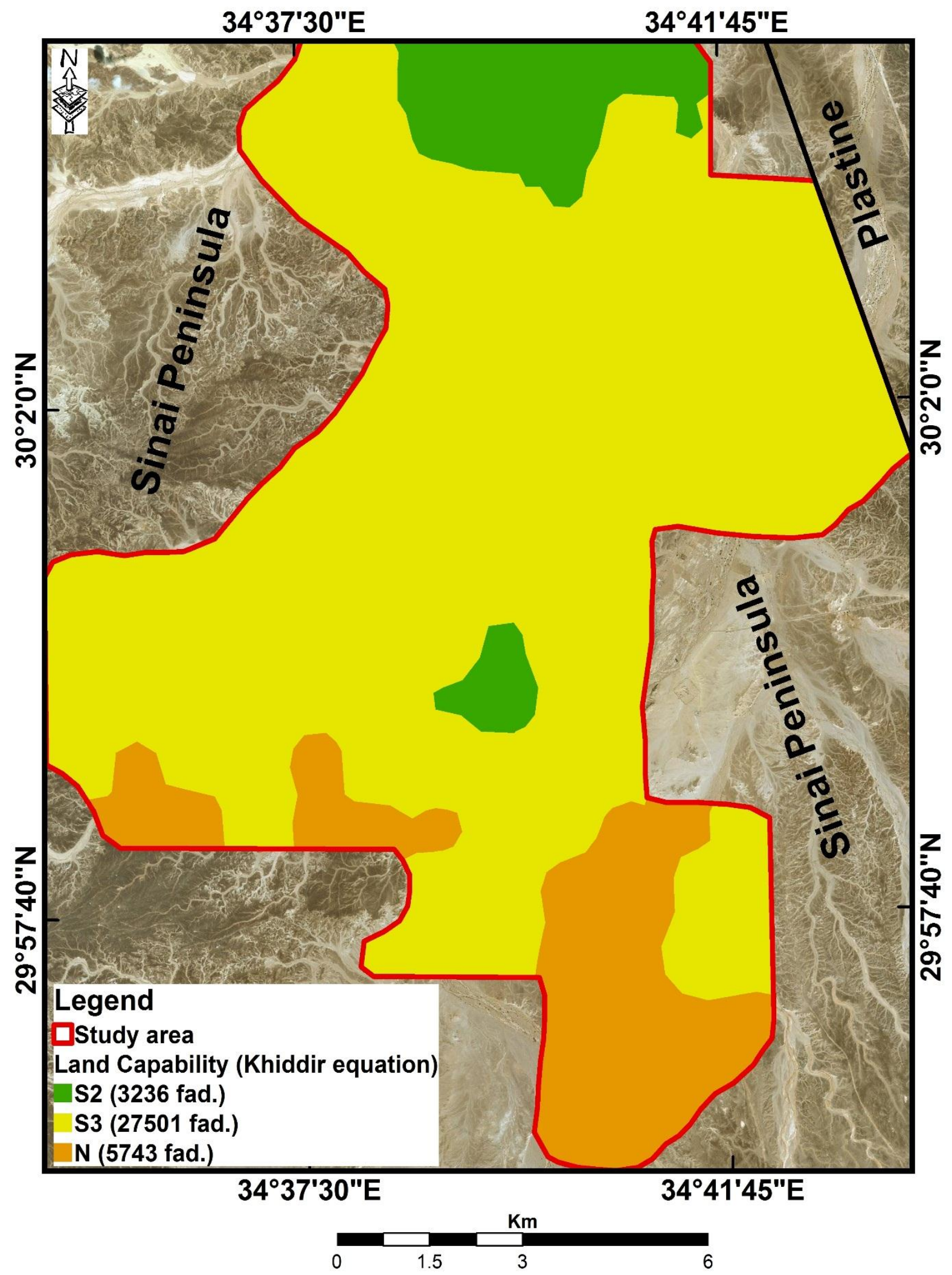

Figure 7. Land capability classes according to Square Root method (Khidder, 1986) 
Table6. Soil properties rates and the final rates of the mapping units (Parametric approach)

\begin{tabular}{|c|c|c|c|c|c|c|c|c|c|c|c|c|c|}
\hline \multirow[t]{2}{*}{ SMU } & \multicolumn{9}{|c|}{ Soil factors rates } & \multicolumn{2}{|c|}{ Storie Index ${ }^{*}$} & \multicolumn{2}{|c|}{ SRM $^{* *}$} \\
\hline & Depth & Gravel & Slope & pH & SAR & EC & Erosion & Texture & drainage & Rate & Grade & Rate & Grade \\
\hline SMU01 & 27.41 & 74.70 & 96.52 & 88.27 & 87.48 & 90.33 & 60.00 & 60.00 & 70.00 & 3.47 & $\mathrm{~N}$ & 9.76 & $\mathrm{~N}$ \\
\hline SMU02 & 63.46 & 96.65 & 96.49 & 88.64 & 83.44 & 67.13 & 60.00 & 60.00 & 90.00 & 12.69 & $\mathrm{~N}$ & 23.90 & $\mathrm{~N}$ \\
\hline SMU03 & 60.13 & 75.59 & 96.41 & 88.49 & 86.12 & 82.45 & 80.00 & 60.00 & 90.00 & 11.89 & $\mathrm{~N}$ & 26.71 & S3 \\
\hline SMU04 & 64.32 & 58.61 & 97.30 & 88.19 & 87.84 & 92.41 & 80.00 & 60.00 & 90.00 & 11.34 & $\mathrm{~N}$ & 25.78 & S3 \\
\hline SMU05 & 86.97 & 76.98 & 96.11 & 88.30 & 88.12 & 94.04 & 100.00 & 60.00 & 100.00 & 28.25 & S3 & 41.17 & S3 \\
\hline SMU06 & 88.71 & 57.33 & 95.34 & 88.19 & 88.30 & 95.11 & 100.00 & 60.00 & 100.00 & 21.55 & $\mathrm{~N}$ & 35.15 & S3 \\
\hline SMU07 & 92.37 & 78.43 & 95.75 & 88.55 & 86.35 & 83.74 & 100.00 & 95.00 & 100.00 & 42.19 & S3 & 57.53 & S2 \\
\hline $\begin{array}{l}\text { S2: Mode } \\
\text { SMU: So } \\
\text { Storie Ind } \\
\text { SRM }^{* *} \text { : M }\end{array}$ & $\begin{array}{l}\text { ly suital } \\
\text { Mapping } \\
\text { Metho } \\
\text { od 2: Sc }\end{array}$ & $\begin{array}{l}\text { S3: } \\
\text { nits } \\
\text { 1: Storie } \\
\text { are Root }\end{array}$ & $\begin{array}{l}\text { ethod } \\
\text { ethod }\end{array}$ & $\begin{array}{l}\text { ording } \\
\text { rding }\end{array}$ & $\begin{array}{l}\text { D'Gee } \\
\text { Khidd }\end{array}$ & $\begin{array}{l}\text { uitable } \\
\text { 008) } \\
\text { al. (19 }\end{array}$ & & & & & & & \\
\hline
\end{tabular}

\section{CONCLUSIONS}

Land evaluation seems to be a profound way to recognize the best agricultural landuses based on assessing the land potentiality or capability. In this sense, wadi Jerafi as one of the most promising area located in Sinai Peninsula especially in North Sinai Governorate was appraised in agricultural point of view using well known land capability classification System MicroLEIS DSS (Cervatana Model) and Revised Storie index. 137 soil profiles were representing the selected study area and their chemical and physical properties were analyzed. Accordingly and specifically by taking soil depth, gravel content and soil texture, seven soil mapping units were delineated. Their chemical and physical properties were statistically described and averaged. The land capability classification either by using Cervatana Model or Revised Storie Index was achieved for the average soil characteristics of each soil mapping units. Accordingly, the study area is covered by two land capability classes as defined by Cervatana Model. These classes are Good suitable (S21), covering the larger area about 23074.85 faddans $(63.25 \%$ of the total area) and Marginally suitable (S31), covering an area about 13405.15 faddans (36.75\% of the total area). Revised Storie index was used to calculate the rate of each soil properties while for assessing the land capability index of each mapping unit two different equations called Storie Method equation and Square Root Method equation were implemented. Accordingly, the Storie Method equation classified the study area into two land capability classes; namely Marginally suitable (S3) covering an area of about 14275 faddans $(39.13 \%$ of the total area and Unsuitable $(\mathrm{N})$ covering an area of about 22205 (60.87\% of the total area). On the other hand, Square Root Method appraised the selected area into 3 suitability classes; Unsuitable $(\mathrm{N})$ covering an area of about 5743 faddans (15.74\% of the total area),
Marginally suitable (S3) covering an area of about 27501 faddans $(75.39 \%$ of the total area) and finally Moderately suitable (S2) covering an area of about 3236 faddans $(8.87 \%$ of the total area.

The study recommended that the modified Storie index model be used as a numerical and non-descriptive method for assessing the physical and chemical properties of soil and applying the Square Root Method (Khiddir et al., 1986) in calculating the capability index of the soil unit. On the other hand, it is not recommended to apply and use the Storie method equation where the reduction of any soil factors in its assessment affects the final assessment of the soil unit. Cervatana model is not recommended for estimating the capability index under the conditions of the Egyptian desert land, as it is semi-quantitative method and it depends on some descriptive characteristics.

\section{ACKNOWLEDGEMENT}

Sincerely, the authors have an honor to appreciate Prof. Dr. Mohamed Abdel Moneam Metwally and Prof. Dr Asmaa Abdu Shata for providing these profound data resulted from the implemented project at Wadi Jerafi, North Sinai Governorate.

\section{REFERENCES}

Abd-El Monsef, H., M. A.-E. R. A.Hassan, and E. Schnug. 2001. Use of remote sensing data and land information system for soil classification. A case study: El-Salam canal in Sinai. Proceeding of the 6th Conf. Geology of Sinai for Development, Ismailia, pp: 283-292.

Addinsoft .2017. XLSTAT Premium version 19.5: A complete statistical add-in for Microsoft Excel.

Al-Busaidi, A., and P.Cookson.2003. Salinity-pH relationships in calcareous soils. Journal of Agricultural and Marine Sciences [JAMS] 8: 41-46.

Aldabaa, A. A. A. 2012. Potential land use in the new reclamation Areas at Wadi Elrayan depression and its 
environs, Elfayum Governorate, Egypt (Doctoral dissertation).

Anderson, J. R. 1976. A land use and land cover classification system for use with remote sensor data.US Government Printing Office.

Atalay, I. 2016. A New approach to the land capability classification: Case study of Turkey. Procedia Environmental Sciences 32:264-274.

De la Rosa, D., M. Anaya-Romero, E. Diaz-Pereira, N. Heredia, and F. J. L. U. P. Shahbazi. 2009. Soil-specific agro-ecological strategies for sustainable land use-A case study by using MicroLEIS DSS in Sevilla Province (Spain). 26: 1055-1065.

De la Rosa, D., F. Mayol, E. Diaz-Pereira, M. Fernandez, and D. de la Rosa Jr.2004. A land evaluation decision support system (MicroLEIS DSS) for agricultural soil protection: With special reference to the Mediterranean region. Environmental Modelling \& Software 19:929-942.

De la Rosa, D., J.Moreno, L.Garcia, and J. Almorza.1992. MicroLEIS: A microcomputer-based Mediterranean land evaluation information system. Soil Use and Management 8:89-96.

Desert Research Center. 2010. Studying the potential of groundwater and soil in Wadi Al-Jerafi, east of central Sinai. Internal report., Unpublished.

EGPC .1987.Geological map of Egypt 1:500,000. NH 36 SE, South Sinai, Egyptian General Petroleum Corperation, Cairo, Egypt : Conoco.

Gabour, T. K. 1998. Land Capability Classification of Northern Sinai Governorate Using GIS. Agricultural Science, Mansoura University 23:5689-5699.

Jahn, R., H.Blume, V. Asio, O.Spaargaren and P.Schad. 2006. Guidelines for soil description, FAO.

Khiddir, S. M., B.Tagliafierro, G.Viccione, O.Ginsburg, R. Badwe, P. Boyle, G. Derricks, A.Dare, T. Evans and A. J. S.Eniu.1986. A statistical approach in the use of parametric systems applied to the FAO framework for land evaluation. 100-250.

Klingebiel, A., and P.Montgomery. 1961. Land-capability classification. Agricultural Handbook. No. 210. Soil Conservation Service. US Department of Agriculture, Washington DC.

Landon, J. R. 2014. Booker tropical soil manual: a handbook for soil survey and agricultural land evaluation in the tropics and subtropics. Routledge.
Mahmoud, H. H., M. S. M.Barseem and A. M. A.Youssef. 2015. Application of the two dimensional geoelectric imaging technique to explore shallow groundwater in wadi El Gerafi basin, Eastern Central Sinai-Egypt. Arabian Journal of Geosciences 8:3589-3601.

O'Geen, A. T. 2008. A revised storie index for use with digital soils information. UCANR Publications.

Rashidi, M., and M. Seilsepour. 2008. Modeling of soil exchangeable sodium percentage based on soil sodium adsorption ratio. International Journal of Agriculture \& Biology 3: 22-6.

Rossiter, D. G. 1996. A theoretical framework for land evaluation. Geoderma 72: 165-190.

Sayed, A. S. A. 2013. Evaluation of the land resources for agricultural development-case study: El-Hammam Canal and its extension, NW Coast of Egypt, Staats- und Universitätsbibliothek Hamburg Germany.

Schoeneberger, P. J. 2012. Field book for describing and sampling soils, Government Printing Office.

Smith, M. 1992. CROPWAT: A computer program for irrigation planning and management, Food \& Agriculture Org.

Soil Science Division Staff 2017. Soil survey manual. C. Ditzler, K. Scheffe, and H.C. Monger (eds.). USDA Handbook 18. Government Printing Office, Washington, D.C.

Storie, R. E. 1978. Storie index soil rating, Division of Agricultural Sciences University of California.

Sys, C., E.Van Ranst and J.Debaveye. 1991a. Land evaluation, Part 1, principles in land evaluation and crop production calculations. International Training Centre for Post-graduate Soil Scientists, University Ghent.

Sys, C., E.Van Ranst, and J. Debaveye. 1991b. Land evaluation, Part 2, principles in land evaluation and crop production calculations. International Training Centre for Post-graduate Soil Scientists, University Ghent.

USDA.2004. Soil survey laboratory methods manual. Soil survey investigations report $\mathbf{4 2}$.

Van Ranst, E., and J. Debaveye.1991. Land Evaluation, Part I: Principles in Land Evaluation and Crop Production Calculations. International Training Center for PostGraduate Soil Scientists, Ghent University, Ghent. 


\section{الملخص العربي}

\section{تقييم القدرة الانتاجية لأراضى حوض وادى الجرافى - شمال سيناء - مصر عماد عبداله محمود ،أحمد سبد أحمدو عبدالصمدعبدالستار علي الضبع}

المدروسة، المعادلة الاولي هي Storie Method و الثانية هي Square Root Method أن منطقة الدراسة قد تميزت إلي درجنين من القدرة الانتاجية و هما هامشية الاصلاحية (S3) و التي تغطي مساحة قدرها

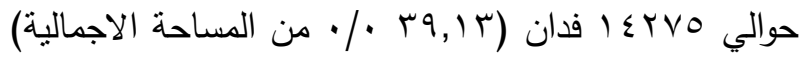
و عديمة الصلاحية (N) و التي تغطي مساحة قدرها حوالي

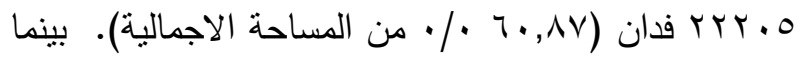
بنطبيق المعادلة الثانية نم رصد ثلاث دراجات من صلاحية التربة و هي علي النحو التالي: عديمة الصلاحية (N) و و

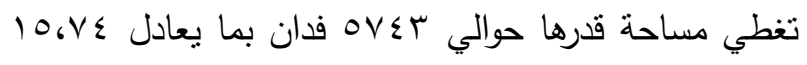
• • من المساحة الاجمالية، هامشية الصلاحية و تغطي

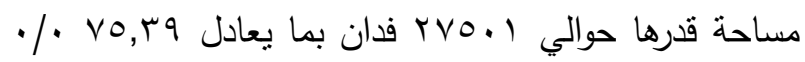
من المساحة الاجمالية، متوسطة الصلاحية و تغطي مساحة

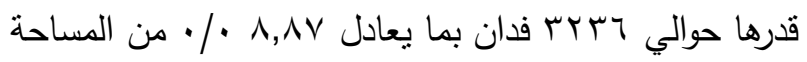
الاجمالية. و خلصت الدراسة إلي أنه يوصي باستخدام نمموذج Storie index المعدل كطريقة رقمية و ليست وصفية لتقييم درجات خصائص التربة الطبيعية و الكيميائية و نطبيق معادلة الجذر التربيعي Square Root Method في حساب القدرة الانتاجية لوحدة التربة الخرائطية. لا ينصح بتطبيق و استخدام معادلة Storie method حيث يؤثر انخفاض أي من

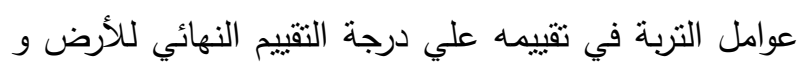
لا ينصح ايضا باستخدام نموذج Cervatana لتقدير القدرة الانتاجية تحت ظروف الاراضي الصحراوية المصرية حيث انه نموذج شبة كمي و يعتمد في تطبيقه علي بعض الصفات
تولي الحكومة المصرية اهنماما كبيرا في الأيام الأخيرة بتتشع المستثمرين على الاستثمار الزراعي في شبه جزيرة سيناء خاصة في القطاع الزراعي. و قد ركزت الدراسة الحالية على تقييم أراضي أحدي المناطق الواعدة في محافظة

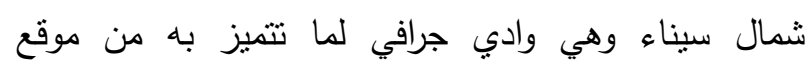
استراتيجي حيث يقع علي الحدود الثرقية مع الأراضي وهي

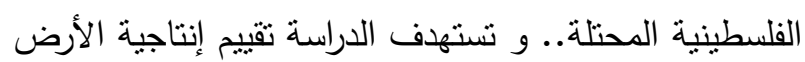
باستخدام نظاميين من النظم المعتمدة دوليا لتقييم التربة و هما Cervatana Model و هevised Storie Index و وبناءً على ذلك، تم إجراء حصر شبكي لأراضي المنطقة محل

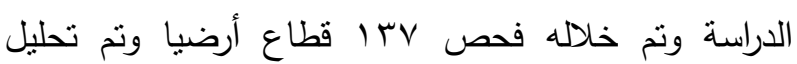
عيناتها تحليلا كيميائياً و طبيعيا. و استتاداً إلى الاختلافات في عمق التربة ومحتوى الحصى و قوام التربة ، تم تمبيز و فصل عدد V وحدات لخرائط التربة و استخدمت النظّ المنوه عنها لتقييم إنتاجيتها أو قدرتها الزراعية. أظهر نموذج Cervatana الأرض الانتاجية وهي S21 (جيدة الصلاحية) و التي تغطي

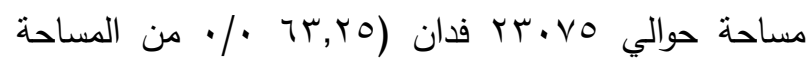
الاجمالية) و S31 (هامشية الصلاحية) و التي تغطي مساحة

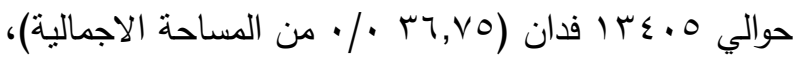
وقد تم رصد أن كل من عمق التربة ومحتوى الحصى و قوام التربة و في بعض الحالات درجة الملوحة هي أكثز العوامل المحددة لاستخدام التربة زراعيا. و من ناحية أخرى، تم لته استخدام مؤشر Storie Index المعدل لتقييم معدلات صفات التربة الطبيعية و الكيميائية المستخدمة في البرنامج و من ثم ثمرنير تطبيق معادلتين مختلفتين لحساب القدرة الانتاجية لمنطقة 